\title{
Analysis on the Impact of Geomagnetic Storm on Reactive Power and Voltage in Extrahigh Voltage Power Grid
}

\author{
Lan $\mathrm{Kang}^{1}{ }^{1}$ Bin $\mathrm{He}^{2}$, Peihong Yang ${ }^{2}$,Xiaoling Dong ${ }^{2}$ \\ School of mining \& coal Engineering, Inner Mongolia University of Science and Technology, Baotou 014010, China; 2. School of \\ Information Engineering, Inner Mongolia University of Science and Technology, Baotou 014010, China )
}

\begin{abstract}
With the growing scale of the power grid and the improvement of the voltage level, the geomagnetically induced currents (GICs) generated by geomagnetic storms has become one of the leading factors that cause large-scale power outages in power grid. Considering the global simultaneity characteristic of geomagnetic storms, this paper presents the concept of mass reactive power disturbance for the first time, analyzes the mechanism of GIC reactive power loss of transformer and reveals the mechanism of mass reactive power disturbance's influence on voltage. Finally, combined with the practical situation of $750 \mathrm{kV}$ EHV power grid in China, the reactive power loss and voltage distribution under the geomagnetic storms at various intensities are calculated, with the grid operating in two typical modes, and the impact of the geomagnetic storm on voltage is illustrated, which provides the theoretical basis for the study of voltage stability.
\end{abstract}

\section{Introduction}

The geomagnetic storm generates a geomagnetically induced current (GIC) in the circuit composed of the transmission line, grounding transformer at both ends and earth ${ }^{[1]}$. When GIC flows through the transformer as a quasi-direct current, it will cause half-wave saturation of the transformer, resulting in a rich harmonic generation of the transformer and a large loss of reactive power ${ }^{[2]}$. A large amount of reactive power has a great impact on the fluctuation of the voltage, which may cause voltage instability or even voltage collapse. If the entire system is not effectively regulated, a large-scale blackout accident will occur ${ }^{[3]}$. Such as the Quebec power grid blackout accident on March 14, 1989; On October 30, 2003, Malmö in northern Sweden also caused a blackout caused by a strong geomagnetic storm [4], furthermore, countries such as China, Brazil, South Africa and Japan have also suffered from different degrees of geomagnetic storms on the grid ${ }^{[5]}$. The effect of geomagnetic storms on reactive power disturbance and voltage is essentially related to the value of GIC. Under the same induced geoelectric fields $(\mathrm{V} / \mathrm{km})$, the GIC of the long-distance high-voltage power grid will be larger ${ }^{[6]}$, which is more likely to cause a large amount of reactive power shortage.

The relationship between GIC and transformer reactive power loss is calculated by the power system analysis synthesis program (PSASP) in [7], the results show that there is a certain linear relationship between the reactive power loss of transformer and the flowing GIC. The transformer magnetization curve is simplified in [8], the transformer reactive power loss model under GIC is constructed, and the corresponding actual circuit is built in the laboratory, the results show that the relationship between reactive power loss of the transformer and GIC is different at various loads. The influence of GIC on voltage stability of power system is preliminarily studied in [9], including static stability and transient stability. The results show that both load model and the intensity of the geomagnetic storm are the factors affecting voltage instability.

With the continuous expansion of voltage grade and scale of the power grid in China, the impact of geomagnetic storms on the voltage stability of the grid should be given enough attention. Northwest power grid is the largest and longest $750 \mathrm{kV}$ EHV interconnected power grid in China. Hence, it is of great significance to study the impact of GIC on Northwest 750kV EHV power grid. Based on the $750 \mathrm{kV}$ EHV power grid in Northwest China, the reactive power loss and voltage distribution of transformers at various geomagnetic storm disturbance (GMD) severity levels is analyzed and the effect of geomagnetic storm on voltage is revealed in this paper. The results provide theoretical basis for voltage stability study at various GMD severity levels.

\section{GIC reactive power loss of the transformer}

\subsection{Mechanism of GIC reactive power loss of the transformer}

When GIC flows through a transformer, DC flux will be generated in the transformer core, the DC flux and AC flux superimpose to form a mixed flux. The saturation

\footnotetext{
*Corresponding author: batefulai558@126.com
} 
degree of the core in half period consistent with the direction of GIC increases greatly, while the flux of the other half cycle is weakened, which is the half-wave saturation. Half-wave saturation flux causes the positive and negative asymmetry of the excitation current. Fig.1 shows the magnetic characteristics of the transformer in half period.

Transformer working in linear region near saturation can improve transformer capacity and efficiency. $\Phi_{0}$ in Fig 1 is the flux extreme value of the linear unsaturated region, the corresponding excitation current is $i_{\mathrm{m} 0}, \Phi_{\mathrm{DC}}$ is the magnetic flux generated by the GIC current, then the mixed flux is:

$$
\Phi=\Phi_{\mathrm{DC}}+\Phi_{0}
$$

Now, the half waveform of the transformer is running in the saturation region, which leads to the distortion of the excitation current waveform and increases the magnitude of the excitation current, as shown in Fig. $i_{m}$.

The reactive power loss of transformer is caused by the excitation current of $90^{\circ}$ lagging system voltage. Transformer reactive power loss is low in normal operation. However, Half-wave saturation caused by GIC makes the excitation current value larger than the normal operation value, which leads to a large increase in the reactive power loss of the transformer. From Fig.1, with the increase of GIC, the half-wave saturation of the transformer increases gradually, which leads to an increase of peak value of excitation current. Hence, the reactive power loss of the transformer is related to the value of GIC, the greater the GIC and the greater the reactive loss of the transformer.

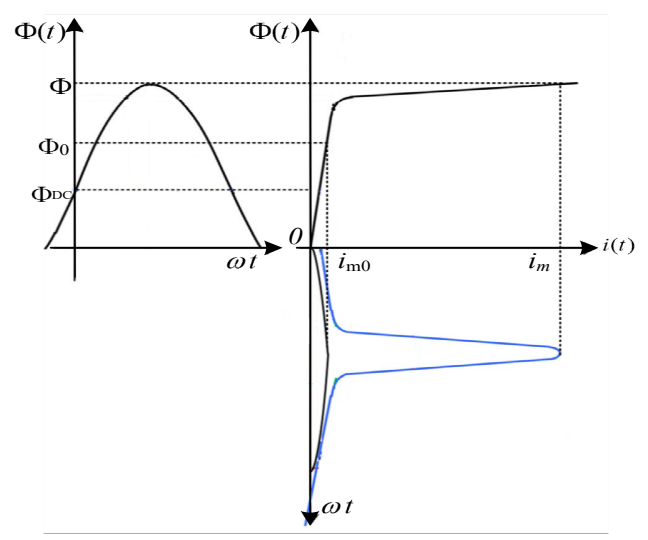

Fig.1 The relationship between excitation current and magnetic flux under GIC

\subsection{Reactive power loss of different types of transformers}

The GIC is a quasi-direct current through the transformer will lead to increased reactive power loss of the transformer. The magnitude of DC flux is related to the structure of transformer, such as the magnetic path provided by transformer. There is no direct path provided for DC flux in the transformer with three-phase and three-column structure, but through air gap with larger reluctance, so the transformer can withstand a large DC bias; The transformer with three-phase and five-column structure can be closed by two side-column cores, so the
DC flux is large and is greatly affected by DC bias.; In single-phase transformers and autotransformers, the DC magnetic path generated by GIC is the same as the main flux, so even a low DC current will produce a large bias, which has the greatest impact on the transformer. The relationship between reactive loss and GIC is described in reference [10]:

$$
Q=k^{*} I_{G I C}+Q_{0}
$$

Where, $Q$ is reactive loss of the transformer; $k$ is the coefficient of reactive loss; $I_{\mathrm{GIC}}$ is the GIC that flowing through the neutral point of the transformer; $Q_{0}$ is the reactive loss during normal operation; The value of $\mathrm{k}$ is: $\mathrm{k} \in[1.18,0.33,0.29,0.66]$ corresponds to the constant coefficients of single-phase transformer, three-phase shell transformer, three-phase three-column core transformer and three-phase five-column transformer, respectively.

It can be seen that the single-phase transformer is most affected by the GIC, the reactive loss under the same GIC is more than three times that of the three-phase shell and the three-column. It can be seen from [11] that the GIC of the EHV long-distance transmission line is larger under the same induced geoelectric fields, the single-phase transformer is basically used in the EHV grid, so that the reactive power loss caused by GIG in EHV power system will be greater, and whether such a large reactive loss will cause voltage stability remains to be further studied.

\section{Analysis of GIC's impact on China's $750 \mathrm{kV}$ power grid}

\subsection{Structure of $750 \mathrm{kV}$ transformer}

Transformers of the $750 \mathrm{kV}$ power grid in northwestern China are single-phase, auto-coupling, three-winding and four-column type. Generally, the high-medium voltage side is star-shaped and the low voltage side is triangular. The high voltage coils are connected in series and the medium and low voltage coils are connected in parallel. The excitation coil and the low voltage coil on the side column are connected in parallel, the voltage regulating coils are connected in series between high-voltage coils and low-voltage coils and adopt medium-voltage voltage regulating mode. And the magnetoresistance in DC magnetic circuit is small, even a small DC current will make the transformer enter half-wave saturation state.

Since the $750 \mathrm{kV}$ transformer is grounded directly and there are DC paths between autotransformers at different voltage levels, GIC will also circulate between the power grids with different voltage levels. The autotransformer of the $750 \mathrm{kV}$ power grid has an electrical connection between the high voltage side and the medium voltage side. Hence, when GIC flows through the neutral point of the transformer, it not only flows through the high voltage winding but also flows through the medium voltage winding for the transmission line of the next voltage level.

\section{2 $750 \mathrm{kV}$ grid reactive power disturbance}

Northwest $750 \mathrm{kV}$ transmission line is the largest and longest transmission system in China's EHV voltage level. 
The wiring diagram is shown in Fig.2. Since the $750 \mathrm{kV}$ line uses 6 split wires, the resistance per unit length is much smaller than the $330 \mathrm{kV}$ line, the length of the $330 \mathrm{kV}$ line is also less than $750 \mathrm{kV}$ line. According to this, the GIC is mainly distributed in the $750 \mathrm{kV}$ power grid. Hence, the $330 \mathrm{kV}$ grid is not considered for loss disturbance and voltage fluctuation.

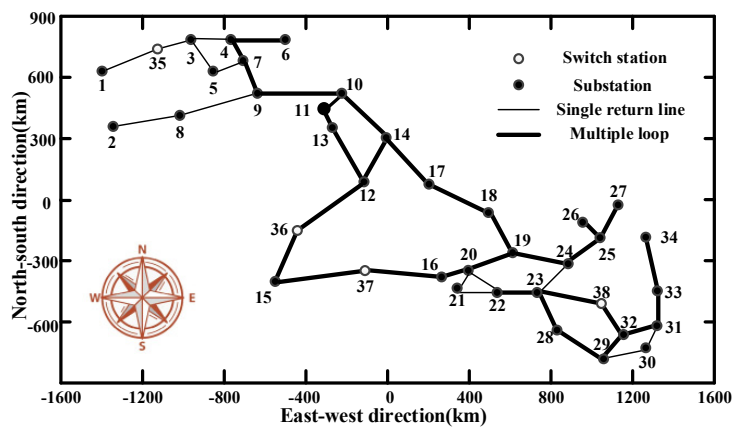

Fig.2 Schematic diagram of the $750 \mathrm{kV}$ transmission system

It can be seen from the above that there is a linear relationship between the reactive power loss generated by the power transformer and the GIC flowing through the single-phase winding of the transformer. Hence, the GIC of the whole system should be calculated before calculating the reactive power loss of the transformer in the transmission system. The calculation method in reference [11] is used in this paper to calculate GIC of the $750 \mathrm{kV}$ transmission system. The induced geoelectric field $1 \mathrm{~V} / \mathrm{km}$ is the interaction of the northward geoelectric fields and the eastward geoelectric fields. The results of the calculation are shown in Fig.3.

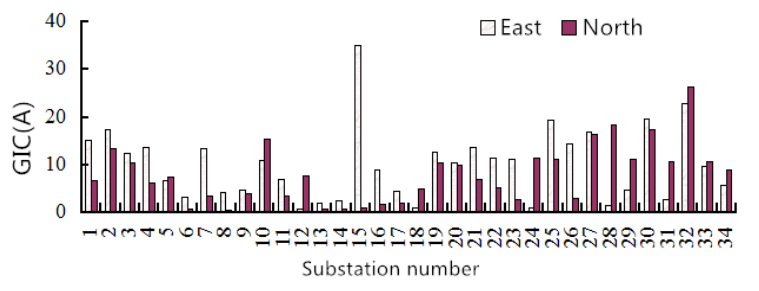

Fig.3 Single-phase GIC value of transformer in the substation

The GIC flowing through each phase winding of the transformer is shown in Fig.3. It is obvious that the GIC in the east direction is greater than the north direction. Moreover, and the larger GIC is mainly distributed in the eastern and western parts of the $750 \mathrm{kV}$ transmission system, the 15th substation has the largest GIC value. According to formula (2), the increase of GIC flowing through transformer will also increase the reactive power loss. The total reactive loss of the system caused by GIC is shown in Table 1.

Table.1 The total reactive power loss of the system under different geoelectric fields directions

\begin{tabular}{cc}
\hline Field direction & Total reactive power loss (MVar) \\
\hline Fast direction & 1209.6 \\
North direction & 959.7 \\
\hline
\end{tabular}

The result is calculated assuming that the uniform geoelectric fields is $E=1 \mathrm{~V} / \mathrm{km}$, only two typical east and north directions are taken. However, it can be seen from the results that there are great differences in reactive power loss at various geoelectric fields. Such a large disturbance of reactive power will cause the fluctuation of voltage. It has a certain impact on the safe and stable operation of the whole power system. There is a certain similarity between the $750 \mathrm{kV}$ power grid in Northwest China and the $735 \mathrm{kV}$ power grid in Quebec, Canada. So it is of certain practical significance to analyze and calculate the voltage fluctuation caused by geomagnetic storms.

\section{$3.3750 \mathrm{kV}$ grid voltage fluctuation}

The reactive power loss $\mathrm{Q}_{\mathrm{GIC}}$ of the transformer caused by GIC means that the reactive power absorbed by the transformer from the power system increases during the geomagnetic storm. Compared with the disturbance without geomagnetic storm, the voltage deviation increases and the voltage fluctuates greatly, which is not conducive to voltage stability. The two-node system is selected to analyze the fluctuation of GIC on the voltage in this paper and shown in Fig.4. Two substations are connected by long-distance transmission lines.

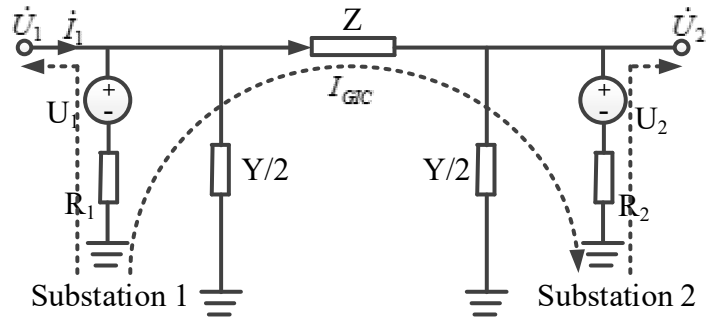

Fig. 4 Line equivalent model during the geomagnetic storm

From the two-node system, $\dot{U}_{2}$ is taken as the reference axis, the line voltage drop is approximately:

$$
\Delta U=\frac{\left(P_{2}+\Delta P\right) R+\left(Q_{2}+Q_{G I C}+Q_{X 2}-Q_{C 2}\right) X}{U_{2}}
$$

Where, $Q_{G I C}$ is the reactive power loss of transformer caused by GIC, $Q_{x 2}$ is the reactive power loss caused by the parallel high voltage reactor. $Q_{C 2}$ is the charging power at the end of the line. $\Delta P$ is active loss of the transformer that caused by GIC-induced transformer reactive loss. From (3), $\triangle P$ can be expressed as:

$$
\Delta P=\frac{\left(2 Q_{2} Q_{G I C}+Q_{G I C}^{2}\right) R}{U_{2}^{2}}
$$

Hence, when the transmission power and parameters of the line remain unchanged, the voltage drop during the geomagnetic storm is only related to $\mathrm{Q}_{\mathrm{GIC}}$. The PSASP software is used to calculate the node voltage in this paper, the East and North geoelectric fields are still taken for analysis and calculation under different loads. According to the allowable deviation of national standard voltage, the allowable deviation of $750 \mathrm{kV}$ voltage is $-5 \% \sim+5 \%$. For this reason, the voltage over-limit index is used to analyze the influence of reactive power loss on voltage fluctuation of power grid in this paper. The over-limit index is represented by the number of node voltage crossing the upper or lower limit, and the calculation results are shown in Table 2. 
Table.2 Substation voltage over-limit

\begin{tabular}{ccc}
\hline Field direction & Large load limit & Small load limit \\
\hline East & 4 & 2 \\
North & 2 & 1 \\
\hline
\end{tabular}

From Table 2, Voltage fluctuation of power grid is greatly affected by geomagnetic storms under large load operation mode. Since the $750 \mathrm{kV}$ power grid structure is mainly east-west, hence, the eastbound geoelectric field has a great impact on the voltage fluctuation of the power grid. Increasing the amplitude of geoelectric field gradually facilitates further analysis of the influence of geomagnetic storms on the voltage fluctuation, and results are shown in Table 3.

Table.3 Substation voltage over-limit in different geoelectric fields

\begin{tabular}{ccccc}
\hline \multirow{2}{*}{ E/V $\mathrm{km}^{-1}$} & \multicolumn{2}{c}{ Large load operation } & \multicolumn{2}{c}{ Small load operation } \\
& East & North & East & North \\
\hline 1 & 4 & 2 & 2 & 1 \\
2 & 4 & 3 & 3 & 2 \\
3 & 5 & 4 & 4 & 2 \\
4 & 7 & 4 & 5 & 4 \\
5 & 12 & 8 & 6 & 4 \\
6 & 18 & 12 & 9 & 7 \\
7 & 22 & 17 & 10 & 9 \\
8 & 28 & 22 & 14 & 12 \\
\hline
\end{tabular}

From Table 3, with the increase of the intensity of geomagnetic storms, the number of over-limit voltage of the power grid also increases, especially when the amplitude of the geoelectric field increases to $8 \mathrm{~V} / \mathrm{km}$. Under the operation mode with a large load of the eastbound geoelectric field, the voltage limit reaches 28 , reaching $82.4 \%$ of the total number of nodes.

\subsection{Results analysis}

According to the analysis and calculation of China's $750 \mathrm{kV}$ actual power grid, geomagnetic storms have a great impact on the reactive power disturbance and voltage fluctuation of the grid. There is a direct relationship between the reactive power disturbance and the intensity of the geomagnetic storm. The stronger the geomagnetic storm is, the more reactive power loss of transformers will be caused, which leads to the greater the reactive power disturbance of the power grid.

\section{Conclusion}

Based on the study of the mechanism of reactive power loss of transformer GIC, the concept of mass reactive power disturbance in power grid is proposed in this paper. Combined with our $750 \mathrm{kV}$ power grid, the reactive power loss and voltage distribution of the power grid at various geomagnetic storm intensities are calculated under two typical operating modes. The results show that the reactive power disturbance is only related to the value of GIC, while the voltage fluctuation is not only related to the value of GIC, but also related to the operation mode of the grid.

Only two kinds of mass geoelectric fields are selected in two typical operation modes to study the reactive power disturbance and voltage fluctuation of transformers caused by GMD in this paper. The harmonic effect of transformer excitation saturation caused by GIC and other equipment failures are not considered which will be the work of further research. Furthermore, the research results of this paper provide theoretical basis for voltage stability research

\section{References}

1. N. Takasu, T. Oshi, F. Miyawaki, et al. An experimental analysis of DC excitation of transformers by geomagnetically induced currents.J. IEEE Trans. Power Del, (1994), 9(2):1173-1183.

2. V.D. Albertson, B.Bozoki, W.E.Feero, et al. Geomagnetic disturbance effects on power systems.J. IEEE Trans. Power Del, (1993),8(3):1206-1216.

3. J. Wen, L.G Liu, S. Xiang, et al. Influences of geomagnetic induced currents on security and stability of power systems.J. Power Syst Technol, (2010), 34(11):24-30

4. M. Wik, A. Viljanen, R. Pirjola, et al. calculaton of geomagnetically induced currents in the $400 \mathrm{kV}$ power grid in southern Sweden.J. Space Wheather, (2008),6(7):11-17.

5. N. Takasu, T. Oshi, F. Miyawaki, et al. An experimental analysis of DC excitation of transformers by geomagnetically induced currents.J. IEEE Trans. Power Del, (1994),9(2):1173-1183.

6. C.M. Liu. Mid-low Latitude Power Grid Geomagnetically Induced Currents and its Assessing Method.D. North China Electric Power University, BeiJing (2009).

7. J. Berge. Impact of geomagnetically induced currents on power transformers.D. University of Western Ontario, Canada, (2011).

8. J. Berge, R.K. Varma, L. Marti. Laboratory validation of the relationship between geomagnetically induced current(GIC) and transformer absorbed reactive power.J. IEEE Electrical Power and Energy Conference, (2011)

9. A. Rezaei-Zare. Behavior of single-phase transformers under geomagnetically induced current conditions.J. IEEE Trans. Power Del, (2014), 29(2): 916-925.

10. X.Z. Dong, Y.L. Liu, J.G.Kappenman. Comparative analysis of exciting current harmonics and reactive power consumption from GIC saturated transformers.J. Power Engineering Society Winter Meeting, 2001 IEEE,(2001), vol.1:318-322.

11. L.M. Liu, S.X. Guo, K. Wei, et al. Calculation of geomagnetically induced currents in interconnected north china-cetral china-east china power grid based on full-node model.J. Power Syst Technol, (2014),38(7):1946-1952. 